\title{
Serviços de atenção ao idoso e estratégias de cuidado domiciliares e institucionais
}

\author{
Aged care services and home health care and institutional strategies
}

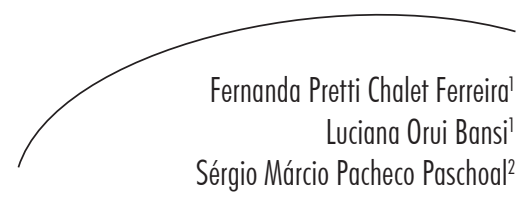

\section{Resumo}

Introdução: As redes de atenção à saúde são fundamentais para assistir a população idosa em seus diversos aspectos. A assistência domiciliar, o Programa Acompanhante de Idosos da Secretaria Municipal da Saúde da cidade de São Paulo e as instituições de longa permanência para idosos compõem as redes de assistência à pessoa idosa. Fazse necessário analisar como a rede entre esses serviços se estabelece e contempla essa demanda de cuidado. Objetivo: Descrever e comparar os serviços assistência domiciliar, Programa Acompanhante de Idosos e instituições de longa permanência para idosos quanto às possíveis diferenças e semelhanças, demanda dos usuários, fluxo assistencial e gestão de serviços. Metodologia: Realizou-se uma revisão bibliográfica não exaustiva da literatura nacional publicada nas bases eletrônicas de dados SciELO e LILACS, em referências literárias e eletrônicas, no período de 1997 a 2012. Resultados: No total, 32 estudos foram incluídos na revisão. Observou-se que a população atendida é predominantemente do sexo feminino, com idade avançada, e recebe assistência multiprofissional. Os serviços de assistência domiciliar e o Programa Acompanhante de Idosos valorizam a manutenção da família e da comunidade como ambiente terapêutico, postergando a institucionalização. A presença do cuidador mostrouse como um dos fatores de maior influência para a permanência dos cuidados domiciliares. Conclusão: Conclui-se que os serviços revisados possuem objetivos que contemplam as demandas dos idosos. Espera-se que a integração dos equipamentos de saúde estabeleça suas ações de acordo com o perfil dos idosos e as características de cuidado oferecidas em cada serviço.

\section{Abstract}

Introduction: Health care networks are critical in assisting the elderly population in several aspects. Home care, the Senior Companion Program [Programa Acompanhante de Idosos] of São Paulo Municipal Health Departament and institutions for the aged compose networks to assist the elderly. It is necessary to analyze how the network is

\footnotetext{
Especialização em Saúde Pública com Ênfase na Estratégia Saúde da Família (ESF). Centro Universitário São Camilo. São Paulo, SP, Brasil.

2 Área Técnica de Saúde da Pessoa Idosa, Coordenadoria da Atenção Básica. Secretaria Municipal da Saúde. São Paulo, SP, Brasil.
}

Palavras-chave: Assistência Domiciliar. Instituição de Longa Permanência para Idosos. Serviços de Saúde para Idosos. Saúde do Idoso. Idoso. Saúde Pública. 
established between these services and features that demand care. Objective: To describe and compare home care services, the Senior Companion Program, and institutions for the aged as to possible differences and similarities, user's demand, flow and care management services. Methods: We carried out a non-exhaustive literature review of national literature published in electronic databases SciELO and LILACS in literary and electronic references in the period from 1997 to 2012. Results: In total, 32 studies were included in the review. It was observed that the served population is predominantly female, with advanced age, and receive multidisciplinary care. Home care services and the Senior Companion Program value the maintenance of family and community as a setting, delaying institutionalization. The presence of the caregiver showed up as one of the most influential factors for the permanence of home care. Conclusion: It is concluded that the revised services have objectives that address the needs of the elderly. It is expected that the integration of health facilities establish their actions according to the profile of the elderly and characteristics of care offered at each service.
Key words: Home Nursing. Homes for the Aged. Health Services for the Aged. Health of the Elderly. Elderly. Public Health.

\section{INTRODUÇÃO}

O crescimento do segmento populacional dos idosos cria uma demanda por serviços médicos e sociais, sendo essencial, para um país em transição demográfica como o Brasil, encontrar alternativas para a tendência de institucionalização de longo prazo dos idosos.

Algumas leis foram criadas para promover ações para a população de idosos do país, como a versão atualizada da Política Nacional de Saúde do Idoso, Portaria no 2.528, de 19 de outubro de 2006. ${ }^{2}$ Esta constitui um marco constitucional histórico, pelo envolvimento de todas as esferas administrativas e governamentais responsáveis pelas ações a serem garantidas à população idosa. ${ }^{1}$

Em 2002, o Ministério da Saúde criou mecanismos para a organização e implantação de Redes Estaduais de Assistência à Saúde do Idoso, ${ }^{3}$ com o objetivo de atender às necessidades dos idosos com qualidade e de forma estruturada para cada nível assistencial. $O$ incentivo à implantação de serviços que possam referenciar as demandas das regiões é fundamental para atingir a população idosa em seus diversos aspectos, através de uma equipe multiprofissional. ${ }^{4}$

As redes estaduais de assistência à saúde do idoso (Portaria GM/MS no 702/2002 e Portaria SAS/MS no 249/2002) são compostas por hospitais gerais e centros de referência em Assistência à Saúde do Idoso. As modalidades que as representam são: internação hospitalar, atendimento ambulatorial especializado, hospitaldia e assistência domiciliar. ${ }^{4}$

A assistência domiciliar (AD), o Programa Acompanhante de Idosos (PAI) da Secretaria Municipal da Saúde da cidade de São Paulo e a instituição de longa permanência para idosos (ILPI) são modalidades de serviços que englobam as redes de assistência ao idoso. De acordo com a mudança do perfil demográfico e das características epidemiológicas encontradas no país, o cuidado domiciliar surgiu como uma modalidade alternativa de atenção à saúde. ${ }^{5}$

A Portaria n n $^{2.527, \text { de }} 27$ de outubro de 2011, redefine a AD no âmbito do Sistema Único de Saúde (SUS), incluindo normas para cadastro desse serviço e sua habilitação, definições, diretrizes, organização domiciliar, modalidades de atenção domiciliar e financiamento. Nessa portaria, consta que a AD é um componente de atenção às urgências, estruturada com as redes de atenção à saúde. ${ }^{6}$

A AD busca garantir a humanização das ações em saúde e a preservação da capacidade funcional do indivíduo, além de sua contextualização em esferas socioculturais, psicológicas e de relações familiares. ${ }^{7}$ Contribui para a otimização dos leitos hospitalares e do atendimento ambulatorial, visando à redução de custos e complicações hospitalares. $^{8}$ 
Desenvolvido pela Secretaria Municipal de Saúde desde 2004, o PAI é uma modalidade de cuidado domiciliar destinado a pessoas idosas em situação de fragilidade e vulnerabilidade social, que se encontram isoladas, sem suporte familiar ou social, sem acesso aos serviços de saúde e com dependência funcional, visando ao provimento do apoio e suporte nas atividades de vida diária (AVDs) e das demais necessidades sociais e de saúde. Esse programa é vinculado a uma unidade de saúde da Rede Básica de Atenção, incluindo Unidade Básica de Saúde (UBS) e Estratégia Saúde da Família (ESF) ou Unidade de Referência à Saúde do Idoso (URSI). ${ }^{9}$

As ILPIs constituem alternativas de cuidados para as pessoas idosas mais frágeis e muito dependentes na execução das tarefas básicas de vida diária e que, por várias razões de ordem médico-sociais, não podem ser mantidas nas suas residências. ${ }^{1}$

Para a Agência Nacional de Vigilância Sanitária (ANVISA), ILPIs são instituições governamentais ou não governamentais, de caráter residencial, destinadas ao domicílio coletivo de pessoas com idade igual ou superior a 60 anos, com ou sem suporte familiar, em condição de liberdade, dignidade e cidadania. São domicílios coletivos que oferecem cuidados e algum tipo de serviço de saúde. Compõe a rede de habitação ${ }^{10}$ e de serviços da assistência social, atendendo às necessidades da comunidade. ${ }^{11}$ No entanto, o envelhecimento da população e o aumento da sobrevivência de pessoas com redução da capacidade física, cognitiva e mental requerem que as instituições deixem de fazer parte apenas da rede de assistência social e integrem a rede de assistência à saúde. ${ }^{10}$

De acordo com o contexto apresentado, faz-se necessário analisar como a rede entre os serviços de AD, PAI e ILPI é estabelecida e se a mesma contempla a demanda de cuidado ao idoso. Portanto, o objetivo deste estudo foi realizar a descrição dos serviços mencionados, comparando-os quanto às possíveis diferenças e semelhanças, demanda dos usuários, fluxo assistencial e gestão de serviços.

\section{METODOLOGIA}

Trata-se de estudo de revisão bibliográfica não exaustiva da literatura nacional publicada nas bases eletrônicas de dados (SciELO e LILACS), em referências literárias e eletrônicas, no período de 1997 a 2012, em português. A busca dos textos foi realizada por duas pesquisadoras, por meio dos seguintes critérios de seleção: busca a partir das palavras-chave, seleção por título, seleção por resumo e leitura dos textos na íntegra.

As palavras-chave utilizadas para a busca nas bases de dados foram: assistência domiciliar OR instituição de longa permanência para idosos OR serviços de saúde para idosos $O R$ saúde do idoso $O R$ saúde do idoso institucionalizado $O R$ acompanhante de idosos.

Foram selecionados os materiais que apresentaram informações descritivas sobre os serviços, associadas ou não às práticas assistenciais prestadas nos mesmos. Os trabalhos foram descritos e posteriormente comparados em relação aos seguintes critérios de análise: características do serviço, perfil da população atendida, presença ou ausência de cuidador, uso de equipamentos da comunidade e do sistema de saúde, composição da equipe multidisciplinar, infraestrutura dos serviços e evolução do usuário nos serviços.

\section{RESULTADOS}

Foram encontrados 271 textos a partir da busca por palavras-chave. Desses, 101 foram selecionados por títulos e 63 após a leitura dos resumos. No total, 57 textos foram lidos na íntegra, sendo 28 sobre o serviço de AD, 25 sobre ILPI e quatro sobre o PAI. Foram excluídos 25 textos por indisponibilidade nos locais de referência ou por não terem relação com os critérios de análise, totalizando 32 incluídos para a descrição dos serviços.

Em relação aos estudos sobre $\mathrm{AD}$, observouse que esta é uma alternativa de cuidado para idosos com incapacidades, doenças crônicas, dificuldades de acesso aos serviços de 
saúde, ${ }^{12}$ em vulnerabilidade social e situação econômica precária, ${ }^{13}$ com sinais de fragilidade e dependência em suas atividades básicas de vida diária (ABVDs) e atividades instrumentais de vida diária (AIVDs). ${ }^{14}$

Os critérios de elegibilidade para o serviço devem visar à equidade entre os pacientes a serem beneficiados e utilizar um instrumento de avaliação que permita a cada membro da equipe interpretar os dados colhidos na triagem. A presença de um cuidador responsável, formal ou informal, é um critério a ser considerado. ${ }^{12}$

A equipe multiprofissional permite $\mathrm{O}$ desenvolvimento e adaptação de funções, favorecendo maior autonomia e independência do paciente. $\mathrm{Na} \mathrm{AD}$, em geral, a equipe é composta por médico, enfermeiro, psicólogo, odontólogo, fonoaudiólogo, fisioterapeuta, terapeuta ocupacional, nutricionista e assistente social. ${ }^{12,14}$ Os serviços de laboratório e radiologia, psicoterapia, oxigenoterapia, equipamentos e serviços de esterilização, farmácia, transporte para pacientes e equipamentos também estão incluídos na assistência ao paciente em domicílio. ${ }^{12}$

Os serviços realizados na AD vão desde cuidados pessoais de suas atividades de vida diária até o uso de alta tecnologia hospitalar e uma rede de apoio para diagnóstico e para outras medidas terapêuticas; além do suporte comunitário e realização de tarefas externas. ${ }^{8} \mathrm{~A}$ visita domiciliar segue vários padrões em seu atendimento, de acordo com o serviço em exercício. ${ }^{14}$

Os resultados dos estudos revisados sobre a AD são apresentados na tabela 1 .

Tabela 1. Apresentação da síntese dos artigos relacionados à assistência domiciliar. São Paulo-SP, 2013.

\begin{tabular}{|c|c|c|}
\hline Autores/Ano & Resultados & Conclusão \\
\hline $\begin{array}{l}\text { Alencar, } \\
\text { Henemann \& } \\
\text { Rothenbuhler, } \\
2008^{15}\end{array}$ & $\begin{array}{l}\mathrm{n}=42 \text {, Curitiba-PR, sexo feminino; } \\
\text { presença de doenças crônico-degenerativas; } \\
\text { cuidadores eram, na maioria, noras e filhas; } \\
\text { presença de equipe multiprofissional; } \\
\text { utilizavam rede de atenção básica; } \\
\text { idosos apresentavam maior dependência } \\
\text { em transferência, andar, banhar-se e } \\
\text { continência; } \\
\text { o atendimento fisioterapêutico } \\
\text { proporcionou melhora da transferência. }\end{array}$ & $\begin{array}{l}\text { A presença e participação dos } \\
\text { cuidadores são fatores centrais para } \\
\text { a execução adequada das orientações } \\
\text { domiciliares. }\end{array}$ \\
\hline $\begin{array}{l}\text { Duca, Thumé \& } \\
\text { Hallal, } 2010^{16}\end{array}$ & $\begin{array}{l}\mathrm{n}=598 \text {, Pelotas-RS, população idosa } \\
\text { masculina, média de idade de 70,4 anos; } \\
\text { incapacidade funcional (ABVD e AIVD) } \\
\text { associada às necessidades de cuidado; } \\
\text { presença de cuidador informal (esposa) na } \\
\text { maioria dos casos. }\end{array}$ & $\begin{array}{l}\text { Resultados associados com maiores } \\
\text { riscos para o desenvolvimento de } \\
\text { fragilidade e maior uso de serviços de } \\
\text { saúde. }\end{array}$ \\
\hline $\begin{array}{l}\text { Thumé et al., } \\
2010^{17}\end{array}$ & $\begin{array}{l}\mathrm{n}=1.593 \text {, Bagé-RS, população de idade } \\
\text { avançada; } \\
\text { utilizavam rede de atenção básica; } \\
\text { presença de doenças crônico-degenerativa, } \\
\text { incapacidades básicas e instrumentais da } \\
\text { vida diária; } \\
\text { presença de equipe multiprofissional. }\end{array}$ & $\begin{array}{l}\text { A satisfação dos usuários da ESF } \\
\text { permitiu diminuir a desigualdade } \\
\text { financeira no acesso aos serviços } \\
\text { de saúde; profissionais devem estar } \\
\text { preparados para a nova realidade } \\
\text { demográfica epidemiológica, incluindo } \\
\text { o domicílio como ambiente terapêutico. }\end{array}$ \\
\hline
\end{tabular}




\begin{tabular}{|c|c|c|}
\hline $\begin{array}{l}\text { Ricci, Kubota \& } \\
\text { Cordeiro, } 2005^{18}\end{array}$ & $\begin{array}{l}\mathrm{n}=40 \text { idosos e seus cuidadores, São Paulo- } \\
\text { SP, maioria do sexo feminino e viúvas, } \\
\text { média de idade de } 80 \text { anos; } \\
\text { parcialmente dependentes; } \\
\text { presença de cuidador informal: filhas. }\end{array}$ & $\begin{array}{l}\text { Serviço permitiu que os cuidadores } \\
\text { fossem bem instruídos quanto } \\
\text { à promoção de saúde de idosos } \\
\text { frágeis. Cuidador deve incentivar a } \\
\text { independência funcional dos pacientes. }\end{array}$ \\
\hline $\begin{array}{l}\text { Bastos, Lemos \& } \\
\text { Mello, } 2007^{19}\end{array}$ & $\begin{array}{l}\mathrm{n}=22 \text {, São Paulo-SP, maioria do sexo } \\
\text { feminino, idade média de } 83 \text { anos, maioria } \\
\text { viúvas; } \\
\text { presença de queda no último ano; } \\
\text { teve episódio de internação antes de } \\
\text { se vincular ao programa de assistência } \\
\text { domiciliar; } \\
\text { presença de cuidador informal e equipe } \\
\text { multiprofissional; } \\
\text { mobilidade comprometida. }\end{array}$ & $\begin{array}{l}\text { O atendimento interdisciplinar alivia a } \\
\text { demanda de idosos que necessitam de } \\
\text { assistência domiciliar, proporcionando } \\
\text { melhor qualidade de vida. }\end{array}$ \\
\hline $\begin{array}{l}\text { Klock, Heck \& } \\
\text { Casarim, } 2005^{20}\end{array}$ & $\begin{array}{l}\text { n=110, Pelotas-RS; } \\
\text { presença de cuidador informal; } \\
\text { utilizavam rede de atenção básica; } \\
\text { presença de equipe multiprofissional; } \\
\text { garantia de acesso à saúde. }\end{array}$ & $\begin{array}{l}\text { A Estratégia Saúde da Família (ESF) } \\
\text { pode contribuir na mudança do } \\
\text { modelo assistencial como alternativa } \\
\text { de assistência à população com } \\
\text { dificuldade de deslocamento aos } \\
\text { serviços de saúde. }\end{array}$ \\
\hline $\begin{array}{l}\text { Aires \& Paz, } \\
2008^{21}\end{array}$ & $\begin{array}{l}\mathrm{n}=176, \mathrm{RS} \text {, maioria do sexo feminino, idade } \\
\text { entre } 60 \text { e } 69 \text { anos; } \\
\text { presença de cuidador informal; } \\
\text { presença de equipe multiprofissional da } \\
\text { ESF; } \\
\text { demanda por programas de orientação da } \\
\text { ESF. }\end{array}$ & $\begin{array}{l}\text { As políticas públicas devem sugerir } \\
\text { e recomendar ações que garantam a } \\
\text { saúde física e mental dos cuidadores } \\
\text { leigos que assumem o cuidado do idoso } \\
\text { dependente. }\end{array}$ \\
\hline $\begin{array}{l}\text { Martins et al., } \\
2009^{22}\end{array}$ & $\begin{array}{l}\mathrm{n}=5 \text { idosos e } 5 \text { familiares, Florianópolis-SC, } \\
\text { maioria do sexo feminino, idade entre } 73 \text { e } \\
88 \text { anos; } \\
\text { presença de doenças crônico-degenerativas; } \\
\text { presença de cuidador informal; } \\
\text { utilizavam rede de atenção básica; } \\
\text { falta de assistência diferenciada no } \\
\text { domicílio. }\end{array}$ & $\begin{array}{l}\text { O Estado deve direcionar ações de } \\
\text { saúde e priorizar atendimento digno } \\
\text { aos idosos para prevenção de doenças, } \\
\text { executando ações que venham resgatar } \\
\text { o completo bem-estar físico, mental, } \\
\text { social e espiritual. }\end{array}$ \\
\hline $\begin{array}{l}\text { Carvalho \& Siefer, } \\
2004^{23}\end{array}$ & $\begin{array}{l}\mathrm{n}=25 \text {, São Paulo-SP, maioria do sexo } \\
\text { masculino, idade entre } 61 \text { e } 70 \text { anos; } \\
\text { presença de cuidador informal; } \\
\text { presença de equipe multiprofissional; } \\
\text { utilizavam rede de atenção secundária; } \\
\text { serviço atende às necessidades de cuidados } \\
\text { paliativos necessários a pacientes com } \\
\text { doenças crônicas e internação de longa } \\
\text { duração; } \\
\text { risco reduzido de infecção hospitalar e } \\
\text { diminuição do número de reinternações. }\end{array}$ & $\begin{array}{l}\text { O serviço proporcionou ao hospital } \\
\text { maior rotatividade do leito e redução } \\
\text { do custo do atendimento por paciente; } \\
\text { o paciente e sua família tiveram } \\
\text { oportunidade de receber atendimento } \\
\text { especializado e com privacidade, } \\
\text { resultando na recuperação mais rápida. }\end{array}$ \\
\hline
\end{tabular}


Sendo o PAI uma alternativa de cuidado domiciliar destinado aos idosos, ele objetiva: proporcionar assistência integral, facilitando o autocuidado, autonomia, independência e melhoria do estado de saúde; evitar ou adiar a institucionalização; reduzir o isolamento social; e capacitar os acompanhantes de idosos em domicílio ou na cidade. ${ }^{9}$

No PAI, a equipe é formada por 17 profissionais: um coordenador (assistente social), um enfermeiro, um médico, dois auxiliares de enfermagem, um motorista, um auxiliar administrativo e dez acompanhantes de idosos (ACIs). As atribuições dos acompanhantes dos idosos estão descritas em um protocolo do programa e são supervisionadas por toda a equipe, que acompanha e avalia suas ações.

No programa, $72 \%$ dos inscritos são mulheres e pelo menos $50 \%$ já estão na oitava década de vida. Em relação às doenças, 32,2\% são afetados por doenças crônicas como hipertensão, diabetes e câncer, e 18,6\% convivem com agravos psíquicos (depressão, demência, ansiedade e síndrome do pânico). ${ }^{24}$

Os acompanhantes do programa oferecem assistência aos idosos frágeis e vulneráveis, com agravos de saúde decorrentes de fragilidade, senilidade, dependência funcional, transtornos mentais, rede social e familiar precárias, com risco de institucionalização; realizam ações de prevenção e promoção à saúde e oferecem companhia em atividades externas. ${ }^{9}$ Nos estudos revisados, verificou-se que as ILPIs são exemplos de suporte não familiar a idosos e estão associadas à assistência social: ${ }^{11}$ emergem como uma alternativa de suporte social para atenção à saúde do idoso, provendo cuidados integrais. ${ }^{25}$

Segundo a Sociedade Brasileira de Geriatria e Gerontologia, ${ }^{26}$ a ILPI é também chamada de abrigo, asilo, lar, casa de repouso, clínica geriátrica ou ancionato. É um estabelecimento para atendimento integral institucional para pessoas com 60 anos de idade ou mais, dependentes ou independentes, que não dispõem de condições para permanecer com a família ou em domicílio.

Nas ILPIs, os idosos podem ser pagantes ou não. $\mathrm{O}$ ambiente deve ser capaz de resgatar antigos hábitos, experiências e recordações, deve ter recursos humanos para atender às necessidades e ser classificado segundo quatro modalidades, de acordo com a especialização do atendimento e a capacidade funcional do idoso. ${ }^{11}$ Os serviços das ILPIs devem contar com assistência médica, odontológica, enfermagem, nutricional, psicológica, farmacêutica, atividades de lazer, reabilitação, serviço social, apoio jurídico e administrativo e serviços gerais. ${ }^{15}$

Hoje a procura de ILPIs é uma questão de saúde pública e surge não só por parte dos idosos com alta dependência, mas também por idosos jovens, entre 60 e 65 anos, independentes, que foram excluídos do mercado de trabalho e da proteção familiar, em decorrência das transformações socioeconômicas em curso na sociedade..$^{25}$

Os resultados dos estudos revisados sobre ILPIs são demonstrados na tabela 2. 
Tabela 2. Apresentação da síntese dos artigos relacionados às instituições de longa permanência para idosos. São Paulo-SP, 2013.

\begin{tabular}{|c|c|c|}
\hline Autores/Ano & Resultados & Conclusão \\
\hline $\begin{array}{l}\text { Camarano \& } \\
\text { Kanso, } 2010^{10}\end{array}$ & $\begin{array}{l}\mathrm{n}=3.295 \text { entidades, Brasil, maioria do sexo } \\
\text { feminino; } \\
\text { dependência funcional ( } 34 \% \text { são independentes); } \\
\text { em todo o território nacional, a maioria das } \\
\text { ILPIs é filantrópica ( } 65,2 \%), 6,6 \% \text { são públicas } \\
\text { e as demais representadas por instituições } \\
\text { religiosas; } \\
\text { serviços oferecidos: assistência médica ( } 66,1 \%) \text {, } \\
\text { fisioterapia }(56 \%) \text {, cursos e atividades de lazer } \\
(50 \%) \text {. }\end{array}$ & $\begin{array}{l}\text { A carência financeira e a falta de } \\
\text { moradia propulsionam a busca das } \\
\text { famílias por ILPIs. }\end{array}$ \\
\hline $\begin{array}{l}\text { Gorzoni \& Pires, } \\
2006^{27}\end{array}$ & $\begin{array}{l}\text { Estudo descritivo, idade média de } 80 \text { anos; } \\
\text { apresentam maior número de doenças físicas, } \\
\text { psíquicas e sociais; } \\
\text { uso do serviço hospitalar nos casos de } \\
\text { complicações; } \\
\text { idosos institucionalizados permanecem mais } \\
\text { tempo em internação e podem apresentar piora } \\
\text { do quadro clínico após alta hospitalar. }\end{array}$ & $\begin{array}{l}\text { As ILPIs devem se responsabilizar } \\
\text { pela detecção precoce e } \\
\text { tratamento de doenças, evitando a } \\
\text { hospitalização. }\end{array}$ \\
\hline Freitas, $2009^{28}$ & $\begin{array}{l}\text { n=7, Bahia; } \\
\text { perfil dos moradores de ILPI: alternativa } \\
\text { para quem vive sozinho; idosos em situação } \\
\text { de abandono; busca por segurança; falta de } \\
\text { condições familiares para cuidar do idoso; } \\
\text { opção aos idosos que não tem filhos; } \\
\text { a ILPI oferece festas, danças, celebração } \\
\text { religiosa, atividade laboral, palestras, gincanas, } \\
\text { bingos e atividades de lazer externas. }\end{array}$ & $\begin{array}{l}\text { As famílias depositam na } \\
\text { instituição a responsabilidade } \\
\text { maior de cuidado de seus parentes; } \\
\text { a equipe não aproxima os } \\
\text { familiares dos idosos, conferindo } \\
\text { mais credibilidade no abandono; } \\
\text { necessidade de capacitação dos } \\
\text { cuidadores e dos profissionais. }\end{array}$ \\
\hline $\begin{array}{l}\text { Pestana \& Espirito } \\
\text { Santo, } 2008^{25}\end{array}$ & $\begin{array}{l}\text { n=17, Rio de Janeiro-RJ; } \\
\text { estudo realizado em uma ILPI filantrópica; } \\
\text { inadequação de infraestrutura, serviço prestado } \\
\text { e recursos humanos; } \\
\text { segundo os idosos, sua saúde é satisfatória e } \\
\text { está relacionada a ausência de dor, desconforto } \\
\text { físico, ausência de doenças, ter autonomia, } \\
\text { lucidez, independência funcional, apoio familiar; } \\
\text { a procura pelo serviço foi feita após a perda } \\
\text { do cônjuge, presença de doenças, condição } \\
\text { financeira insuficiente, falta de condiçoes } \\
\text { familiares para acolher e cuidar os idosos } \\
\text { (espaço, recurso ou estrutura); } \\
\text { os idosos não realizavam atividades fora da } \\
\text { instituição. }\end{array}$ & $\begin{array}{l}\text { As políticas destinadas à saúde } \\
\text { da pessoa idosa precisam } \\
\text { oferecer alternativas para evitar } \\
\text { a institucionalização através } \\
\text { dos espaços de promoção de } \\
\text { saúde, mantendo a autonomia e } \\
\text { qualidade de vida do idoso; as } \\
\text { estratégias devem ser estabelecidas } \\
\text { por equipes multiprofissionais } \\
\text { capacitadas que possam associar } \\
\text { a participação familiar em suas } \\
\text { ações. }\end{array}$ \\
\hline
\end{tabular}


Perlini, Leite \& $\quad \mathrm{n}=6$ cuidadores, Ijuí-RS;

Furini, $2007^{29} \quad$ perfil dos moradores de ILPIs: dificuldade para realizar as AVDs, gerenciar seu domicílio e finanças; presença de doenças crônicas incapacitantes;

filhos, cônjuges ou parentes enxergam a institucionalização como alternativa de cuidado; relacionamentos familiares conturbados pregressos também estão associados à institucionalização;

idosos que tomam a decisão de se institucionalizar têm condição financeira suficiente para se sentir acolhido na instituição.

Ximenes \& Corte, Estudo de caso em ILPI filantrópica e religiosa $2006^{30}$ de São Paulo; presença de equipe multiprofissional; alta rotatividade de profissionais sem gestão adequada gerando desorganização; ambiente de abandono e tristeza; idosos sem vontade de viver.
As políticas públicas destinadas ao idoso devem priorizar a manutenção dessa população em seus domicílios, apoiando as famílias, educando-as e atendendo a suas demandas de cuidado com profissionais qualificados.

\begin{abstract}
A ILPI precisa investir na qualificação de profissionais; necessidade interligada ao trabalho em equipe; o conhecimento dos residentes, bem como a escuta dos mesmos proporciona um "fazer" com significado, passível de modificações diárias, segundo a autonomia dos sujeitos.
\end{abstract}

Os autores elaboraram um manual

Costa, $2004^{31} \quad$ Estudo de caso em uma ILPI, Guarulhos-SP, maioria do sexo feminino, idade média de 84,64 anos;

$17,39 \%$ independentes, $25 \%$ parcialmente dependentes, 57,26\% dependentes; $44,57 \%$ com família nuclear, $50 \%$ recebem visita constantemente;

presença de equipe multiprofissional;

a ILPI oferece atividades de trabalhos manuais, exercício físico, atividades espirituais, comemorações, musicoterapia, leitura, passeios; cursos para cuidadores de idosos, centro de convivência, centro-dia, oficina de arte, parque, lanchonete, setor de voluntário.

Lenardt, Michel \&'Tallmann, $2009^{32}$ $\mathrm{n}=20$, Curitiba-PR, população idosa feminina, média de idade acima de 80 anos;

independentes para a maior parte das atividades de vida diária;

maior risco de quedas devido à presença concomitante de múltiplos fatores de risco; as idosas realizavam atividades fora $\mathrm{da}$ instituição para encontro social;

a ILPI oferece oportunidade de atualização por meio da leitura;

prática de exercícios físicos;

presença de equipe multiprofissional;

baixa prevalência de indicadores de depressão e elevada prevalência de indicadores de demência.
Os aspectos da satisfação pessoal poderão ser promovidos trazendo contribuição positiva para a sensação de bem-estar, incentivando e facilitando o engajamento dos idosos em atividades que promovam o envolvimento com a família e a comunidade. 


\begin{tabular}{|c|c|c|}
\hline $\begin{array}{l}\text { Castro, Jordão \& } \\
\text { Maia, 201033 }\end{array}$ & $\begin{array}{l}\text { n= } 66, \mathrm{RS} \text {, população masculina, idade entre } 50 \\
\text { e } 90 \text { anos; } \\
\text { maioria portadora de alguma deficiência física } \\
\text { ou mental; } \\
\text { pouca comunicação entre os internos e espera } \\
\text { da morte. }\end{array}$ & $\begin{array}{l}\text { Necessidade e importância de } \\
\text { traçar dispositivos para a criação } \\
\text { de espaços terapêuticos que } \\
\text { possam gerar novos modos de } \\
\text { sociabilidade. }\end{array}$ \\
\hline Camarano, $2007^{34}$ & $\begin{array}{l}\mathrm{n}=49 \text { instituições, Norte do Brasil, maioria do } \\
\text { sexo masculino e independentes; } \\
\text { presença de equipe multiprofissional. }\end{array}$ & $\begin{array}{l}\text { Na região em questão, há baixa } \\
\text { proporção de idosos residindo nas } \\
\text { ILPIs devido a reduzida oferta de } \\
\text { serviços, altos custos, preconceitos, } \\
\text { estereótipos e preferência do } \\
\text { cuidado por familiares. }\end{array}$ \\
\hline $\begin{array}{l}\text { Telles Filho \& } \\
\text { Petrilli Filho, } \\
2002^{35}\end{array}$ & $\begin{array}{l}\text { n=12, interior de São Paulo-SP, maioria do sexo } \\
\text { feminino, idade entre } 60 \text { e } 91 \text { anos; } \\
\text { presença de doenças crônico-degenerativas; } \\
\text { condições precárias de saúde, idade avançada, } \\
\text { distúrbios de comportamento, incapacidade } \\
\text { de exercer atividades laborais, dificuldade } \\
\text { financeira, falta de respaldo familiar, } \\
\text { necessidade de reabilitação, ausência temporária } \\
\text { de cuidador domiciliar, estágios terminais de } \\
\text { patologias, dependência elevada; } \\
\text { presença de equipe multiprofissional. }\end{array}$ & $\begin{array}{l}\text { Sugestões às instituições: plantas } \\
\text { físicas adequadas para suprir as } \\
\text { reais necessidades dos usuários, } \\
\text { programação de atividades, } \\
\text { profissionais especializados, } \\
\text { promoção da independência } \\
\text { e estímulo à participação da } \\
\text { família e cuidadores; alternativas } \\
\text { de assistência para não } \\
\text { institucionalização: hospitais-dia, } \\
\text { centros de convivência e serviços } \\
\text { de enfermagem domiciliares. }\end{array}$ \\
\hline $\begin{array}{l}\text { Fernandes \& } \\
\text { Almeida, } 2001^{36}\end{array}$ & $\begin{array}{l}\text { n=39, João Pessoa-PB, estudo realizado em ILPI } \\
\text { filantrópica; } \\
\text { a maioria dos idosos possuía deficiências } \\
\text { cognitivas graves; } \\
\text { a instituição é um ambiente favorecedor das } \\
\text { mudanças fisiológicas, conflitos psicológicos, } \\
\text { distúrbio do sono e julgamento alterado, que } \\
\text { podem ocorrer devido ao isolamento. }\end{array}$ & $\begin{array}{l}\text { Necessidade de reorganização } \\
\text { das políticas públicas brasileiras } \\
\text { que favoreçam a implantação } \\
\text { de medidas holísticas capazes } \\
\text { de proporcionar melhoria da } \\
\text { qualidade de vida dos idosos. }\end{array}$ \\
\hline
\end{tabular}

\section{DISCUSSÃO}

A comparação entre os serviços de $\mathrm{AD}$, ILPI e PAI mostra que a maioria da população atendida é predominantemente do sexo feminino e idade avançada. Em relação ao perfil dos idosos atendidos, na $\mathrm{AD}$, a maioria apresenta doenças crônico-degenerativas e funcionalidade comprometida, associadas à presença de cuidador (familiares). A maioria apresenta história prévia de internação hospitalar.

Nas ILPIs, os idosos apresentam doenças físicas, psíquicas, crônico-degenerativas, vulnerabilidade social, condições precárias de saúde, estágios terminais de patologias, deficiências cognitivas graves e funcionalidade comprometida. A procura da instituição, em geral filantrópica, acontece em situações nas quais o idoso vive sozinho, abandonado, em busca por segurança, por falta de condições familiares para o cuidado, e é uma opção ao idoso que não tem filhos. Geralmente é feita após a perda do cônjuge, presença de doenças e condição financeira insuficiente.

Em comparação com o PAI, os idosos são afetados por doenças crônicas como hipertensão arterial, diabetes e câncer, e uma parcela convive com agravos psíquicos (depressão, demência, 
ansiedade e síndrome do pânico). Assim como na AD e ILPI, encontram-se em situação de fragilidade clínica e dependência funcional.
Com o objetivo de ilustrar o fluxo assistencial do idoso com diferentes demandas, segue-se esquema representativo.

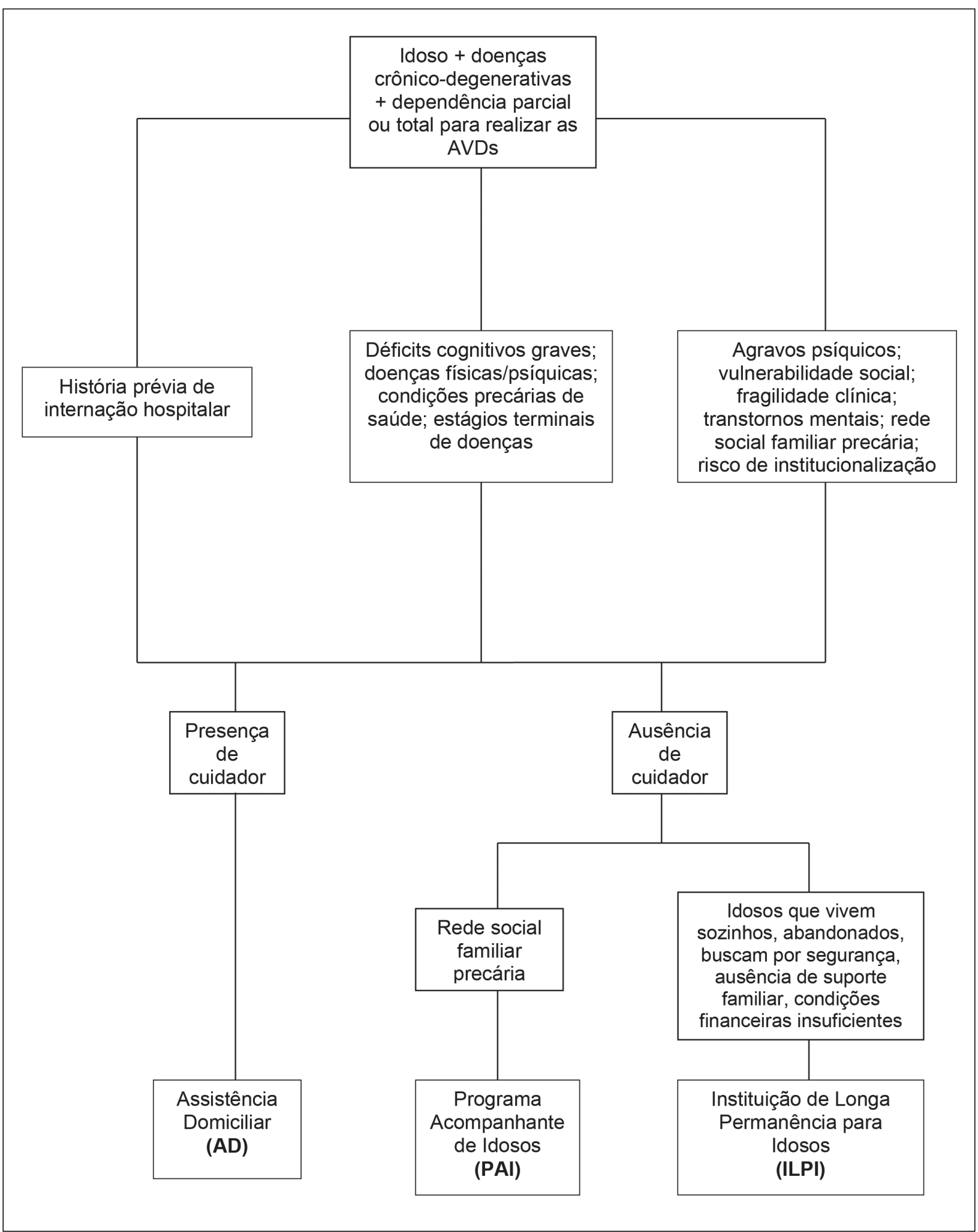

Figura 1. Fluxo assistencial. São Paulo-SP, 2013. 
Através do fluxo assistencial (figura 1), observa-se que um dos fatores de maior influência para a permanência dos cuidados domiciliares, principalmente na $\mathrm{AD}$, é a presença de um cuidador, que em geral é a esposa; em segundo lugar, a filha mais velha casada, em seguida, a filha viúva ou solteira. ${ }^{37}$

Para que os cuidados sejam bem desempenhados nos domicílios, o preparo e a escolha adequada do cuidador são fundamentais, e isso requer boa estrutura familiar. Atualmente, o número de idosos cuidando de idosos é crescente, sendo que muitas vezes os próprios cuidadores apresentam doenças potencialmente incapacitantes. ${ }^{38}$

O processo de educação e orientação dos cuidadores de idosos deve estar presente nos serviços de assistência domiciliar. Martins et al. ${ }^{39}$ identificaram quais as necessidades de cuidado e educação apresentadas por seis cuidadores de idosos de um serviço de assistência domiciliar no estado de Santa Catarina. A partir das entrevistas, os cuidadores apresentaram interesse na aquisição de orientações relacionadas às doenças e cuidados de saúde. Nenhuma cuidadora possuía formação para cuidar de idosos e isso fomentou a busca por metodologias de educação em saúde por parte dos profissionais. As informações sobre os cuidados devem ser disseminadas e distribuídas pelo serviço público desde a atenção primária, a fim de auxiliar os profissionais e pacientes durante todo o processo de cuidado.

Relacionada com as ações primárias em saúde, a ESF possui o objetivo de reorganizar o modelo tradicional da atenção básica, unindo promoção, proteção e recuperação da saúde, usando a integração familiar como principal ferramenta. ${ }^{40-43}$ É um instrumento para a implantação de programas com foco nos cuidadores, uma vez que, por sua proximidade com o território e com a comunidade, tem uma visão mais ampla do contexto de vida cotidiana desses idosos. ${ }^{42}$
De acordo com os estudos revisados, os níveis de atenção básica e secundária são utilizados pelos usuários da AD e PAI, garantindo-lhes o acesso à saúde e inserindo-os em fluxos assistenciais em diversas modalidades de serviço. Na ILPI, os idosos fazem uso do serviço hospitalar nos casos de complicações, privados e do SUS, e realizam atividades fora da instituição para encontro social ou comunitário.

Em comum, os usuários da AD e ILPI deveriam contar com os cuidados do enfermeiro, médico, auxiliar de enfermagem, assistente social, fisioterapeuta, nutricionista, psicólogo, terapeuta ocupacional e farmacêutico. Exclusivamente na AD, fonoaudiólogo e agente comunitário de saúde complementam a equipe. Nem todos os autores descreveram a composição multiprofissional, porém destacaram a importância da mesma.

No estudo de Lenardt, Michel \& Tallmann, ${ }^{32}$ a ILPI se diferencia pela presença do médico geriatra, odontologista, musicoterapeuta, cuidador de idosos (contratado pela ILPI), oferta de serviços de limpeza, lavanderia, manutenção, zeladoria, cozinha e setor administrativo. No PAI, o acompanhante de idosos recebe suporte contínuo da equipe técnica (assistente social, médico e enfermeiro), responsável pela elaboração e revisão do plano de cuidado individual, implementado pelo acompanhante no domicílio e nas atividades externas programadas. Há discussão de casos periodicamente entre o acompanhante e a equipe técnica do programa. Compreende-se que esses serviços são vantajosos por valorizarem a manutenção da família e da comunidade no ambiente terapêutico, postergando a institucionalização. ${ }^{44}$

A partir da análise de dados do trabalho com grupos focais, Batista ${ }^{45}$ analisou, em sua dissertação de mestrado, a opinião de 11 acompanhantes de idosos (ACIs) de 12 equipes do PAI, a respeito do processo de trabalho desenvolvido no programa. Verificou-se que os ACIs não tinham formação anterior específica ao trabalho do PAI e havia pouca experiência 
no trabalho com idosos. A regulamentação da profissão seria um respaldo para suas atribuições, além das elencadas pelo Documento Norteador. $^{44}$ Os ACIs apresentaram forte vínculo com os idosos e relataram satisfação com o trabalho desempenhado, mesmo encontrando demandas imprevistas além dos cuidados em saúde. Adicionalmente, o trabalho foi considerado local de aprendizado, devido à troca de experiência com os colegas de profissão, com o próprio idoso e seus familiares. Em concordância com o pioneirismo do programa, os acompanhantes facilitam a inserção social dos idosos em situações de vulnerabilidade, os quais reconhecem a importância do seu trabalho. ${ }^{44}$

No entanto, contradizendo o que foi encontrado nos estudos revisados, a realidade das condições das ILPIs foi retratada pela Comissão dos Direitos Humanos da Câmara dos Deputados ${ }^{46}$ em 2002, quando organizaram a V Caravana Nacional de Direitos Humanos. A comissão visitou, durante uma semana, 28 ILPIs de quatro estados brasileiros (São Paulo, Rio de Janeiro, Recife e Paraná). Segundo os autores, além da irregularidade de funcionamento, muitas instituições funcionavam em condições de precariedade e com cuidados de saúde insuficientes. A maioria dos idosos era dependente, passava grande parte do tempo sem atividades e sem privacidade. O relatório enfatizou a necessidade de mudança no perfil institucional dos estabelecimentos que prestam cuidados aos idosos no Brasil, os quais não deveriam ser a última opção de assistência aos idosos dependentes.

Segundo a RDC/ANVISA n ${ }^{\circ} 283,{ }^{47}$ em relação ao funcionamento da ILPI, esta deverá contar com um responsável técnico pelo serviço com formação de nível superior, não especificando que o mesmo deva ter conhecimento na área de saúde ou gestão em saúde. $\mathrm{O}$ documento não preconiza os cuidados multiprofissionais, como descrito nos artigos.

Diferentemente do modelo de AD desempenhado em outros serviços, sobretudo nos particulares, na atenção básica as visitas domiciliares são frequentes nos casos de idosos acamados e dependentes. O papel da ESF, com ênfase na atenção ao idoso, se destaca pela identificação dos agravos mais frequentes e sua intervenção. ${ }^{40}$ No entanto, as ações de promoção e prevenção em saúde precisam ser intensificadas para cumprir o princípio da integralidade, um dos pilares do SUS.

Em estudo de revisão integrativa, Carvalho, Assunção \& Bocchi $^{48}$ avaliaram a percepção dos profissionais quanto ao atendimento da ESF ao idoso. Os autores observaram que alguns fatores dificultam a relação da ESF com idosos, incluindo o ineficaz planejamento do serviço, irregularidade de assistência das equipes e reduzida capacitação profissional. Os autores também concluíram que os profissionais ainda não trabalham a favor das reflexões referentes à assistência do idoso, uma vez que a ESF tem transformado o modelo de atenção básica.

A ESF e o Núcleo de Apoio à Saúde da Família (NASF) são representados por diversas categorias profissionais que complementam as ações desempenhadas pelos profissionais do PAI. Essas ações interdisciplinares em saúde, assim como a capacitação desses profissionais, são importantes para garantir a qualidade e a integralidade do cuidado ao idoso assistido em domicílio.

Para ilustrar a infraestrutura e evolução dos usuários nos serviços, segue-se esquema representativo (figura 2). 


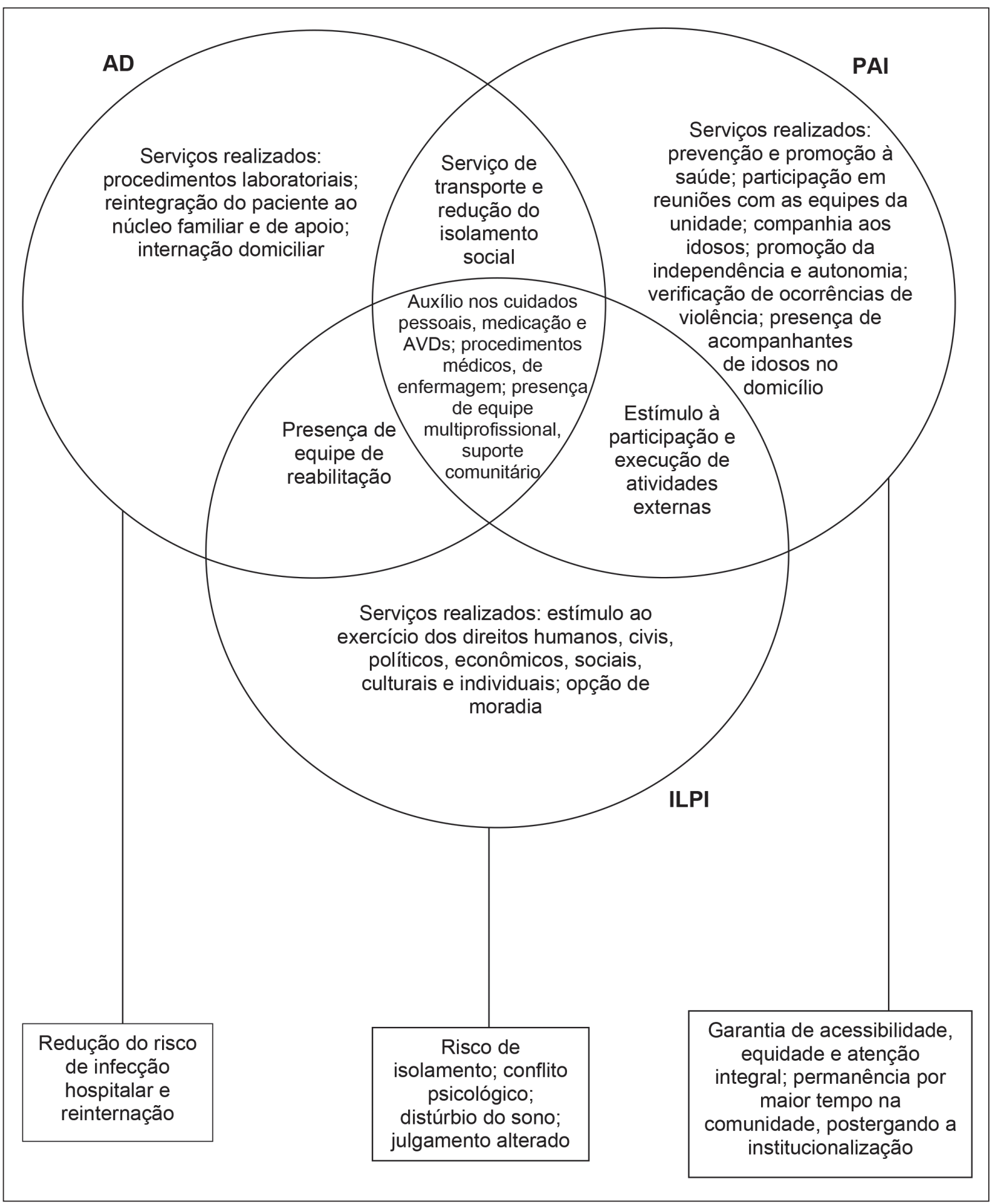

Figura 2. Infraestrutura e evolução dos usuários nos serviços. São Paulo-SP, 2013. 
Verifica-se que a ILPI e o PAI possuem a iniciativa de promover a inclusão dos idosos em atividades a favor do envelhecimento ativo, mesmo naqueles que apresentam ou têm chances de desenvolver fragilidade clínica.

Em relação à evolução do usuário nos serviços, o risco de infecção hospitalar e o número de reinternações são reduzidos na AD. A ILPI pode ser um ambiente favorecedor das mudanças decorrentes do isolamento. As causas associadas ao isolamento são atribuídas a ausência da família, perda das referências e escassez das visitas ao longo do tempo. ${ }^{49}$ Embora os estudos revisados destaquem essas questões, esse modelo de moradia oferece oportunidades de socialização entre os residentes, os quais apresentam características em comum e podem realizar trocas de experiências de vida, motivando-se uns aos outros.

O PAI tem se revelado como estratégia de garantia da acessibilidade, equidade e atenção integral às pessoas idosas em situação de fragilidade. ' É um programa de fácil tecnologia social e favorece a implantação em outras localidades, com base em seu Documento Norteador ${ }^{44}$ permitindo que as pessoas permaneçam pelo maior tempo na comunidade exercendo seus direitos de cidadania.?

As principais limitações desta pesquisa estiveram relacionadas com a dificuldade em encontrar estudos científicos sobre o PAI, possivelmente por se tratar de um programa novo, e a impossibilidade de acesso a alguns artigos em bibliotecas de outros estados do Brasil.

\section{CONCLUSÃO}

De acordo com os objetivos propostos, foi observado que nos serviços de assistência domiciliar e no Programa Acompanhante de Idosos a presença do cuidador e do acompanhante de idoso, respectivamente, são fatores de proteção. Para que os cuidados sejam bem desempenhados, o preparo e a escolha adequada do cuidador são fundamentais.

Constatou-se que a demanda por cuidados institucionais tende a crescer, devido ao envelhecimento populacional e mudanças nos arranjos familiares. A ILPI é um serviço que deveria oferecer diversas atividades a seus residentes, promovendo melhora da autoestima e qualidade de vida.

As equipes multiprofissionais são fundamentais para o funcionamento dos serviços descritos. Os profissionais devem estar capacitados para ofertar cuidados de acordo com a demanda dos idosos e com o tipo de serviço proposto, principalmente para a população longeva e frágil, usuária dos serviços revisados.

Um modelo de atenção à saúde do idoso que pretenda apresentar efetividade e eficiência necessita de ações que envolvam todos os níveis e possuir um fluxo delineado de ações de educação, promoção da saúde, prevenção de doenças, postergação de moléstias e reabilitação de agravos.

Com base na presente revisão, esperase que a integração dos equipamentos de saúde, disponíveis no sistema de referência e contrarreferência, estabeleça suas ações de acordo com o perfil dos idosos e as características de cuidado oferecidas em cada serviço. Novos estudos devem ser realizados para facilitar a reflexão dos profissionais e gestores de saúde sobre o tema em questão. 


\section{REFERÊNCIAS}

1. Brito FC, Ramos LR. Serviços de atenção à saúde do idoso. In: Papaléo Netto, M. Tratado de Gerontologia. 2. ed. São Paulo: Atheneu; 2007. p. 671-679.

2. Brasil. Portaria n ${ }^{\circ}$ 2.528, de 19 de Outubro de 2006. Aprova a Política Nacional de Saúde da Pessoa Idosa. Portal da Saúde. 2006. Disponível em: http:// portal.saude.gov.br/portal/arquivos/pdf/2528\%20 aprova $\% 20 \mathrm{a} \% 20$ politica $\% 20$ nacional $\% 20 \mathrm{de} \% 20$ saude $\% 20 \mathrm{da} \% 20$ pessoa $\% 20$ idosa.pdf

3. Brasil. Mistério da Saúde.Redes Estaduais de Atenção à Saúde do Idoso: guia operacional e portarias relacionadas [Internet]. Brasília, DF: Ministério da Saúde; 2002 [Acesso em 2011 ago 12]. (Série A. Normas e manuais técnicos). Disponível em http://bvsms.saude.gov.br/bvs/publicacoes/ redesestaduais.pdf.

4. Louvison MCP, Barros S. Políticas públicas e envelhecimento: a construção de uma política de direitos e os desafios da atenção integral à saúde da pessoa idosa no SUS. BIS, Bol Inst Saúde 2009;(47):53-5.

5. Duarte YAO, Diogo MJD. Atendimento Domiciliar: um enfoque gerontológico. São Paulo: Atheneu; 2000.

6. Brasil. Ministério da Saúde. Portaria no 2.527 de 27 de outubro de 2011. Redefine a atenção domiciliar no âmbito do Sistema Único de Saúde (SUS). 2011. Disponível em: ftp://ftp.saude.sp.gov.br/ftpsessp/ bibliote/informe_eletronico/2011/iels.out.11/ Iels205/U_PT-MS-GM-2527_271011.pdf.

7. Rehem TCMSB, Trad LAB. Assistência domiciliar em saúde: subsídios para um projeto de atenção básica brasileira. Ciênc Saúde Coletiva 2005;10Supl:231-42.

8. Floriani CA, Schramm FR. Atendimento domiciliar ao idoso: problema ou solução? Cad Saúde Pública 2004;20(4):986-94.

9. Berzins MAVS, Paschoal SMP. Programa acompanhante de idosos. BIS, Bol Inst Saúde 2009;(47):53-5.

10. Camarano AA, Kanso S. As instituições de longa permanência para idosos no Brasil. Rev Bras Estud Popul 2010;27(1):232-5.

11. Born T, Boechat NS. A qualidade dos cuidados ao idoso institucionalizado. In: Freitas EV, Py L, Cançado FAX, Doll J, Gorzoni ML, organizadores. Tratado de geriatria e gerontologia. 2. ed. Rio de Janeiro: Guanabara Koogan; 2006. p. 1131-43.

12. Carletti SM, Rejani MI. Atenção domiciliária ao paciente idoso. In: Papaléo Netto M. Gerontologia. São Paulo: Atheneu;1996. p. 415-430.
13. Maximino VS. Terapia ocupacional e atenção domiciliar nos processos de envelhecimento. Mundo Saúde 2005;29(4):523-7.

14. Beendendo RS, Gurgel JM. Interdisciplinaridade na assistência domiciliária. In: Papaléo Netto M. Tratado de Gerontologia. São Paulo: Atheneu; 2007. p. 701-9.

15. Alencar MCB, Henemann L, Rothenbuhler R. A capacidade funcional de pacientes e a fisioterapia em um programa de assistência domiciliar. Fisioter Mov 2008;21(1):11-20.

16. Del Duca GF, Thumé E, Hallal PC. Prevalência e fatores associados ao cuidado domiciliar a idosos. Rev Saúde Pública 2011;45(1):113-20.

17. Thumé E, Augusto Facchini AL, Tomasi E, Vieira LAS. Assistência domiciliar a idosos: fatores associados, características do acesso e do cuidado. Rev Saúde Pública 2010;44(6):1-10.

18. Ricci NA, Kubota MT, Cordeiro RC. Concordância de observações sobre a capacidade funcional de idosos em assistência domiciliar. Rev Saúde Pública 2005;39(4):655-62.

19. Bastos CC, Lemos ND, Mello AN. Perfil clínicodemográfico dos pacientes inseridos em um programa de assistência domiciliária no município de São Paulo. Rev Kairós 2007;10(2):205-24.

20. Klock AD, Heck RM, Casarim ST. Cuidado domiciliar: a experiência da residência multiprofissional em Saúde da Família/UFPEL-MS/ BID. Texto \& Contexto Enferm 2005;14(2):237-45.

21. Aires M, Paz AA. Necessidades de cuidado aos idosos no domicílio no contexto de estratégia de saúde da família. Rev Gaúcha Enferm 2008;29(1):83-9.

22. Martins JJ, De Jesus JM, Do Nascimento ERP, Erdmann AL, Candemil MC, Belaver GM. O cuidado no contexto domiciliar: o discurso de idosos/familiares e profissionais. Rev Enferm UERJ 2009;17(4):556-62.

23. Carvalho TA, Siefer MW. Pesquisa de opinião com usuários de serviços de assistência domiciliar contratados no pós-alta hospitalar (home care). Rev Soc Cardiol Estado de São Paulo 2004;14(5):10-8.

24. Prefeitura de São Paulo, Secretaria da Saúde. Programa acompanhante de idosos é ampliado na capital [Internet]. São Paulo: Prefeitura de São Paulo; 2012 [acesso em 25 Fev 2012]. Disponível em: http://extranet.saude.prefeitura.sp.gov.br/noticias/ programa-de-acompanhantes-de-idosos-e-ampliadona-capital. 
25. Pestana LC, Espirito Santo FH. As engrenagens da saúde na terceira idade: um estudo com idosos asilados. Rev Esc Enferm USP 2008;42(2):268-75.

26. Sociedade Brasileira De Geriatria e Gerontologia. Instituição de longa permanência para idosos: manual de funcionamento. São Paulo: SBGG; 2004

27. Gorzoni ML, Pires SL. Idosos asilados em hospitais gerais. Rev Saúde Pública 2006;40(6):1124-30.

28. Freitas AVS. Por trás dos muros: um estudo sobre a vida de idosos em instituição de longa permanência [tese]. Salvador: Universidade Federal da Bahia, Departamento de Instituto de Saúde Coletiva; 2009.

29. Perlini NMOG, Leite MT, Furini AC. Em busca de uma instituição para a pessoa idosa morar: motivos apontados por familiares. Rev Esc Enferm USP 2007;41(2):229-36.

30. Ximenes MA, Corte B. O fazer institucionalizado: o cotidiano do asilamento. Rev Kairós 2006;9(2):135-45.

31. Costa RCD. Modelo metodológico organizacional aplicável a gestão de instituições de longa permanência para idosos [tese]. São Paulo: Faculdade de Medicina da Universidade de São Paulo; 2004.

32. Lenardt MH, Michel T,Tallmann AEC. A condição de saúde de idosas residentes em instituição de longa permanência. Cogitare Enferm 2009;14(2):227-36.

33. Castro GD, Jordão AB, Maia GF. Ampliando a clínica com idosos institucionalizados. Rev Mal-estar Subj 2010;10(1):193-210.

34. Camarano AA. Características das instituições de longa permanência para idosos: região Norte. Brasília: IPEA; 2007.

35. Telles Filho PCP, Petrilli Filho JF. Causas da inserção de idosos em uma instituição asilar. Esc Anna Nery Rev Enferm 2002;6(1):135-43.

36. Fernandes MGM, Almeida CB. Processo de pensamento alterado em idosos na vivência asilar. Nursing 2001;4(34):22-5.

37. Rodrigues SLA, Watanabe HAW, Derntl AM. A saúde de idosos que cuidam de idosos. Rev Esc Enferm USP 2006;40(4):493-500.

38. Karsch UM. Idosos dependentes: famílias e cuidadores. Cad Saúde Pública 2003;19(3):861-66.

39. Martins JJ, De Albuquerque GL, Do Nascimento ERP, Barra DCC, De Souza WGA, Pacheco WNS. Necessidades de educação em saúde dos cuidadores de pessoas idosas no domić́lio. Texto \& contexto Enferm 2007;16(2):254-62.
40. Silvestre JAN, Costa Neto MM. Abordagem do idoso em programas saúde da família. Cad Saúde Pública 2003;19(3):839-47.

41. Brasil. Ministério da Saúde, Secretaria de Atenção a Saúde, Departamento de Atenção Básica. Departamento de Atenção a Saúde. Política Nacional de Atenção Básica [Internet]. Brasília, DF: Ministério da Saúde; 2006. [acesso em 21 Jul 2012]. (Série Pactos pela Saúde, vol. 6). Disponível em: http://sistemas. aids.gov.br/forumprevencao_final/sites/default/files/ arquivos/Politica_Nacional_Nac._de_At._Basica.pdf.

42. Bufon MCM, Rodrigues CK. A saúde da família como enfoque estratégico para a organização da atenção básica primária em saúde. Visão acad 2005;6(2):96-8

43. Pavarini SCI, Luchesi BM, Fernandes HCL, Mendiondo MSZ, Filizola CLA, Barham EJ, et al. Genograma: avaliando a estrutura familiar de idosos de uma unidade de saúde da família. Rev Eletrônica Enferm 2008;10(1):39-50.

44. São Paulo. Secretaria Municipal da Saúde, Coordenação de Atenção Básica, Área Técnica de Saúde da Pessoa Idosa. Documento norteador do Programa Acompanhante de Idosos do Município de São Paulo. São Paulo: Secretaria Municipal de Saúde; 2012.

45. BATISTA MPP. Reflexões sobre o processo de trabalho do acompanhante de idosos do Programa Acompanhante de Idosos (PAI) no município de São Paulo, SP (Brasil) [dissertação]. São Paulo: Faculdade de Medicina, Universidade de São Paulo; 2013.

46. Brasil. Câmara dos Deputados, Comissão de Direitos Humanos. Relatório da $5^{\text {a }}$ Caravana Nacional De Direitos Humanos: uma amostra da realidade dos abrigos e asilos de idosos no Brasil. Brasília, DF: Câmara dos Deputados; 2002.

47. Brasil.Resolução RDC no 283, de 26 de setembro de 2005. Aprova o Regulamento Técnico que define normas de funcionamento para as Instituições de Longa Permanência para Idosos. Diário Oficial da União. 2005.

48. Carvalho CJA, Assunção RC, Bocchi SCM. Percepção dos profissionais que atuam na Estratégia de Saúde da Família quanto a assistência prestada aos idosos: revisão integrativa de literatura. Physis 2010;20(4):1307-24.

49. Leite VL, Santana RF. Intervenções psicoeducativas para famílias de idosos institucionalizados. In: Associação Brasileira de Enfermagem. SENPE 2011. Anais do $16^{\circ}$ Seminário Nacional de Pesquisa em Enfermagem; 19-22 Jun 2011; Campo Grande, MS: ABEn; 2011. p. 1165-8. 